\title{
On quaternions with generalized Fibonacci and Lucas number components
}

Emrah Polatli* and Seyhun Kesim

"Correspondence: emrah.polatli@beun.edu.tr Department of Mathematics, Bulent Ecevit University, Zonguldak, 67100, Turkey

\begin{abstract}
In this paper, we give the exponential generating functions for the generalized Fibonacci and generalized Lucas quaternions, respectively. Moreover, we give some new formulas for binomial sums of these quaternions by using their Binet forms.
\end{abstract}

MSC: $11 \mathrm{~B} 39$

Keywords: generalized Fibonacci quaternions; generalized Lucas quaternions

\section{Introduction}

The well-known Fibonacci and Lucas sequences are defined by the following recurrence relations: for $n \geq 0$,

$$
F_{n+2}=F_{n+1}+F_{n}
$$

and

$$
L_{n+2}=L_{n+1}+L_{n}
$$

where $F_{0}=0, F_{1}=1, L_{0}=2$, and $L_{1}=1$, respectively. Here, $F_{n}$ is the $n$th Fibonacci number and $L_{n}$ is the $n$th Lucas number.

The Binet formulas for the Fibonacci and Lucas sequences are given by

$$
F_{n}=\frac{\alpha^{n}-\beta^{n}}{\alpha-\beta}
$$

and

$$
L_{n}=\alpha^{n}+\beta^{n},
$$

where

$$
\alpha=\frac{1+\sqrt{5}}{2} \text { and } \beta=\frac{1-\sqrt{5}}{2} .
$$

The numerous relations connecting Fibonacci and Lucas numbers have been given by Vajda [1].

(c) 2015 Polatli and Kesim. This article is distributed under the terms of the Creative Commons Attribution 4.0 International License (http://creativecommons.org/licenses/by/4.0/), which permits unrestricted use, distribution, and reproduction in any medium, provided you give appropriate credit to the original author(s) and the source, provide a link to the Creative Commons license, and indicate if changes were made. 
Carlitz, Ferns, Layman and Hoggatt studied many properties of binomial sums of these numbers (see [2-5]).

The generalized Fibonacci and Lucas sequences, $\left\{U_{n}\right\}$ and $\left\{V_{n}\right\}$, are defined by the following recurrence relations: for $n \geq 0$ and any nonzero integer $p$,

$$
U_{n+2}=p U_{n+1}+U_{n}
$$

and

$$
V_{n+2}=p V_{n+1}+V_{n},
$$

where $U_{0}=0, U_{1}=1, V_{0}=2$, and $V_{1}=p$, respectively. If we take $p=1$, then $U_{n}=F_{n}$ (nth Fibonacci number) and $V_{n}=L_{n}$ ( $n$th Lucas number).

Let $\lambda$ and $\mu$ be the roots of the characteristic equation $x^{2}-p x-1=0$. Then the Binet formulas for the sequences $\left\{U_{n}\right\}$ and $\left\{V_{n}\right\}$ are given by

$$
U_{n}=\frac{\lambda^{n}-\mu^{n}}{\lambda-\mu}
$$

and

$$
V_{n}=\lambda^{n}+\mu^{n}
$$

where $\lambda=\left(p+\sqrt{p^{2}+4}\right) / 2$ and $\mu=\left(p-\sqrt{p^{2}+4}\right) / 2$.

From these equalities, by putting $\Delta=p^{2}+4$, we see that $1+\lambda^{2}=\lambda \sqrt{\Delta}$ and $1+\mu^{2}=$ $-\mu \sqrt{\Delta}$.

The generating function and the exponential generating function of a sequence $\left\{a_{n}\right\}$ are defined by

$$
a(x)=\sum_{n=0}^{\infty} a_{n} x^{n}
$$

and

$$
\hat{a}(x)=\sum_{n=0}^{\infty} a_{n} \frac{x^{n}}{n !}
$$

respectively.

Now, we would like to explain how the generating function of a given sequence $\left\{a_{n}\right\}$ is derived: Firstly, we write down a recurrence relation which is a single equation expressing $a_{n}$ in terms of other elements of the sequence. This equation should be valid for all integers $n$, assuming that $a_{-1}=a_{-2}=\cdots=0$. Then we multiply both sides of the equation by $x^{n}$ and sum over all $n$. This gives, on the left, the sum $\sum_{n=0}^{\infty} a_{n} x^{n}$, which is the generating function $a(x)$. The right-hand side should be manipulated so that it becomes some other expression involving $a(x)$. Lastly, if we solve the resulting equation, we get a closed form for $a(x)$. For more details and properties related to the generating functions and special functions, we refer to [6-12]. 
A quaternion $p$ with real components $a_{0}, a_{1}, a_{2}, a_{3}$, and basis $\mathbf{1}, \mathbf{i}, \mathbf{j}, \mathbf{k}$ is an element of the form

$$
p=a_{0} \mathbf{1}+a_{1} \mathbf{i}+a_{2} \mathbf{j}+a_{3} \mathbf{k}=\left(a_{0}, a_{1}, a_{2}, a_{3}\right) \quad\left(a_{0} \mathbf{1}=a_{0}\right)
$$

where

$$
\begin{aligned}
& \mathbf{i}^{2}=\mathbf{j}^{2}=\mathbf{k}^{2}=-\mathbf{1}, \\
& \mathbf{i j}=\mathbf{k}=-\mathbf{j i}, \quad \mathbf{j} \mathbf{k}=\mathbf{i}=-\mathbf{k} \mathbf{j}, \quad \mathbf{k} \mathbf{i}=\mathbf{j}=-\mathbf{i} \mathbf{k} .
\end{aligned}
$$

Throughout this paper, we work in the real division quaternion algebra without specific references being given.

The $n$th Fibonacci and the $n$th Lucas quaternions were defined by Horadam in [13] as

$$
Q_{n}=F_{n}+F_{n+1} \mathbf{i}+F_{n+2} \mathbf{j}+F_{n+3} \mathbf{k}
$$

and

$$
K_{n}=L_{n}+L_{n+1} \mathbf{i}+L_{n+2} \mathbf{j}+L_{n+3} \mathbf{k},
$$

respectively, where $F_{n}$ and $L_{n}$ are the $n$th Fibonacci number and the $n$th Lucas number.

Similar to Horadam, the $n$th generalized Fibonacci quaternion and $n$th generalized Lucas quaternion are defined by

$$
\mathcal{Q}_{n}=U_{n}+U_{n+1} \mathbf{i}+U_{n+2} \mathbf{j}+U_{n+3} \mathbf{k}
$$

and

$$
\mathcal{K}_{n}=V_{n}+V_{n+1} \mathbf{i}+V_{n+2} \mathbf{j}+V_{n+3} \mathbf{k},
$$

respectively [14]. Here, $U_{n}$ and $V_{n}$ are the $n$th generalized Fibonacci number and the $n$th generalized Lucas number.

For $n \geq 0$, the following recurrence relations hold:

$$
\mathcal{Q}_{n+2}=p \mathcal{Q}_{n+1}+\mathcal{Q}_{n}
$$

and

$$
\mathcal{K}_{n+2}=p \mathcal{K}_{n+1}+\mathcal{K}_{n}
$$

where

$$
\begin{aligned}
& \mathcal{Q}_{0}=\left(0,1, p, p^{2}+1\right), \quad \mathcal{Q}_{1}=\left(1, p, p^{2}+1, p^{3}+2 p\right) \\
& \mathcal{K}_{0}=\left(2, p, p^{2}+2, p^{3}+3 p\right), \quad \mathcal{K}_{1}=\left(p, p^{2}+2, p^{3}+3 p, p^{4}+4 p^{2}+2\right) .
\end{aligned}
$$


In [15], Iakin gave the Binet formulas for the generalized Fibonacci and generalized Lucas quaternions as follows:

$$
\mathcal{Q}_{n}=\frac{\lambda \lambda^{n}-\underline{\mu} \mu^{n}}{\lambda-\mu}
$$

and

$$
\mathcal{K}_{n}=\underline{\lambda} \lambda^{n}+\underline{\mu} \mu^{n}
$$

where $\underline{\lambda}=1+\lambda \mathbf{i}+\lambda^{2} \mathbf{j}+\lambda^{3} \mathbf{k}$ and $\underline{\mu}=1+\mu \mathbf{i}+\mu^{2} \mathbf{j}+\mu^{3} \mathbf{k}$.

Iyer [16] gave some relations connecting the Fibonacci and Lucas quaternions. In [17], Swamy derived the relations of generalized Fibonacci quaternions. Iakin [18, 19] introduced the concept of a higher order quaternion and generalized quaternions with quaternion components. Horadam [20] studied the quaternion recurrence relations. In [21], Halici derived generating functions and many identities for Fibonacci and Lucas quaternions. In [22], Flaut and Shpakivskyi investigated some properties of generalized Fibonacci quaternions and Fibonacci-Narayana quaternions. Very recently, Ramirez [14] has obtained some combinatorial properties of the $k$-Fibonacci and the $k$-Lucas quaternions.

Inspired by these results, in the present paper we give the exponential generating functions for the generalized Fibonacci and generalized Lucas quaternions. Moreover, we derive some new formulas for binomial sums of these quaternions by using their Binet forms.

\section{Some properties of generalized Fibonacci and Lucas quaternions}

Theorem 2.1 ([14]) The generating functions for the generalized Fibonacci and generalized Lucas quaternions are

$$
F(t)=\frac{\mathcal{Q}_{0}+\left(\mathcal{Q}_{1}-p \mathcal{Q}_{0}\right) t}{1-p t-t^{2}}
$$

and

$$
L(t)=\frac{\mathcal{K}_{0}+\left(\mathcal{K}_{1}-p \mathcal{K}_{0}\right) t}{1-p t-t^{2}}
$$

respectively.

Proof Let $F(t)=\sum_{n=0}^{\infty} \mathcal{Q}_{n} t^{n}$ and $L(t)=\sum_{n=0}^{\infty} \mathcal{K}_{n} t^{n}$. Then we get the following equation:

$$
\begin{aligned}
& \sum_{n=0}^{\infty} \mathcal{Q}_{n} t^{n}-p t \sum_{n=0}^{\infty} \mathcal{Q}_{n} t^{n}-t^{2} \sum_{n=0}^{\infty} \mathcal{Q}_{n} t^{n} \\
& \quad=\mathcal{Q}_{0}+\left(\mathcal{Q}_{1}-p \mathcal{Q}_{0}\right) t+\sum_{n=2}^{\infty}\left(\mathcal{Q}_{n}-p \mathcal{Q}_{n-1}-\mathcal{Q}_{n-2}\right) t^{n}
\end{aligned}
$$

Since, for each $n \geq 2$, the coefficient of $t^{n}$ is zero in the right-hand side of this equation, we obtain

$$
F(t)=\frac{\mathcal{Q}_{0}+\left(\mathcal{Q}_{1}-p \mathcal{Q}_{0}\right) t}{1-p t-t^{2}}
$$


Similarly, we obtain

$$
L(t)=\frac{\mathcal{K}_{0}+\left(\mathcal{K}_{1}-p \mathcal{K}_{0}\right) t}{1-p t-t^{2}} .
$$

Theorem 2.2 The exponential generating functions for the generalized Fibonacci and generalized Lucas quaternions are

$$
\sum_{n=0}^{\infty} \frac{\mathcal{Q}_{n}}{n !} t^{n}=\frac{\lambda e^{\lambda t}-\underline{\mu} e^{\mu t}}{\lambda-\mu}
$$

and

$$
\sum_{n=0}^{\infty} \frac{\mathcal{K}_{n}}{n !} t^{n}=\underline{\lambda} e^{\lambda t}+\underline{\mu} e^{\mu t}
$$

Proof By the Binet formula for the generalized Fibonacci quaternions, we get

$$
\begin{aligned}
\sum_{n=0}^{\infty} \frac{\mathcal{Q}_{n}}{n !} t^{n} & =\sum_{n=0}^{\infty}\left(\frac{\underline{\lambda} \lambda^{n}-\mu \mu^{n}}{\lambda-\mu}\right) \frac{t^{n}}{n !} \\
& =\frac{\underline{\lambda}}{\lambda-\mu} \sum_{n=0}^{\infty} \frac{(\lambda t)^{n}}{n !}-\frac{\underline{\mu}}{\lambda-\mu} \sum_{n=0}^{\infty} \frac{(\mu t)^{n}}{n !} \\
& =\frac{\underline{\lambda} e^{\lambda t}-\underline{\mu} e^{\mu t}}{\lambda-\mu} .
\end{aligned}
$$

Similarly, by the Binet formula for the generalized Lucas quaternions we obtain

$$
\begin{aligned}
\sum_{n=0}^{\infty} \frac{\mathcal{K}_{n}}{n !} t^{n} & =\sum_{n=0}^{\infty}\left(\underline{\lambda} \lambda^{n}+\underline{\mu} \mu^{n}\right) \frac{t^{n}}{n !} \\
& =\underline{\lambda} \sum_{n=0}^{\infty} \frac{(\lambda t)^{n}}{n !}+\underline{\mu} \sum_{n=0}^{\infty} \frac{(\mu t)^{n}}{n !} \\
& =\underline{\lambda} e^{\lambda t}+\underline{\mu} e^{\mu t} .
\end{aligned}
$$

Now, we give the following theorems in which the first formulas are proved, and the remaining formulas can be obtained similarly.

Theorem 2.3 For $n, k \geq 0$, we have

$$
\begin{aligned}
& \sum_{i=0}^{n}\left(\begin{array}{l}
n \\
i
\end{array}\right) \mathcal{Q}_{2 i+k}= \begin{cases}\Delta^{\frac{n}{2}} \mathcal{Q}_{n+k} & \text { if } n \text { is even, } \\
\Delta^{\frac{n-1}{2}} \mathcal{K}_{n+k} & \text { if } n \text { is odd, }\end{cases} \\
& \sum_{i=0}^{n}\left(\begin{array}{l}
n \\
i
\end{array}\right) \mathcal{K}_{2 i+k}= \begin{cases}\Delta^{\frac{n}{2}} \mathcal{K}_{n+k} & \text { if } n \text { is even, } \\
\Delta^{\frac{n+1}{2}} \mathcal{Q}_{n+k} & \text { if } n \text { is odd, }\end{cases} \\
& \sum_{i=0}^{n}\left(\begin{array}{c}
n \\
i
\end{array}\right)(-1)^{i} \mathcal{Q}_{2 i+k}=(-p)^{n} \mathcal{Q}_{n+k}, \\
& \sum_{i=0}^{n}\left(\begin{array}{c}
n \\
i
\end{array}\right)(-1)^{i} \mathcal{K}_{2 i+k}=(-p)^{n} \mathcal{K}_{n+k} .
\end{aligned}
$$


Proof If we use the Binet formula for the generalized Fibonacci quaternions, we get

$$
\begin{aligned}
\sum_{i=0}^{n}\left(\begin{array}{l}
n \\
i
\end{array}\right) \mathcal{Q}_{2 i+k} & =\sum_{i=0}^{n}\left(\begin{array}{l}
n \\
i
\end{array}\right)\left(\frac{\underline{\lambda} \lambda^{2 i+k}-\underline{\mu} \mu^{2 i+k}}{\lambda-\mu}\right) \\
& =\frac{\underline{\lambda} \lambda^{k}}{\lambda-\mu} \sum_{i=0}^{n}\left(\begin{array}{l}
n \\
i
\end{array}\right) \lambda^{2 i}-\frac{\mu}{\lambda-\mu} \sum_{i=0}^{k}\left(\begin{array}{l}
n \\
i
\end{array}\right) \mu^{2 i} \\
& =\frac{\underline{\lambda} \lambda^{k}}{\lambda-\mu}\left(1+\lambda^{2}\right)^{n}-\frac{\mu \mu^{k}}{\lambda-\mu}\left(1+\mu^{2}\right)^{n} \\
& =\frac{\underline{\lambda} \lambda^{k}}{\lambda-\mu}(\lambda \sqrt{\Delta})^{n}-\frac{\mu}{\lambda-\mu}(-\mu \sqrt{\Delta})^{n} \\
& = \begin{cases}\Delta^{\frac{n}{2}} \mathcal{Q}_{n+k} & \text { if } n \text { is even, } \\
\Delta^{\frac{n-1}{2}} \mathcal{K}_{n+k} & \text { if } n \text { is odd. }\end{cases}
\end{aligned}
$$

Theorem 2.4 ([14]) For $n \geq 0$, we have

$$
\begin{aligned}
& \sum_{i=0}^{n}\left(\begin{array}{l}
n \\
i
\end{array}\right) p^{i} \mathcal{Q}_{i}=\mathcal{Q}_{2 n}, \\
& \sum_{i=0}^{n}\left(\begin{array}{l}
n \\
i
\end{array}\right) p^{i} \mathcal{K}_{i}=\mathcal{K}_{2 n} .
\end{aligned}
$$

Proof By the Binet formula for the generalized Fibonacci quaternions, we have

$$
\begin{aligned}
\sum_{i=0}^{n}\left(\begin{array}{l}
n \\
i
\end{array}\right) p^{i} \mathcal{Q}_{i} & =\sum_{i=0}^{n}\left(\begin{array}{l}
n \\
i
\end{array}\right) p^{i}\left(\frac{\underline{\lambda} \lambda^{i}-\underline{\mu} \mu^{i}}{\lambda-\mu}\right) \\
& =\frac{\underline{\lambda}}{\lambda-\mu} \sum_{i=0}^{n}\left(\begin{array}{l}
n \\
i
\end{array}\right)(p \lambda)^{i}-\frac{\underline{\mu}}{\lambda-\mu} \sum_{i=0}^{n}\left(\begin{array}{l}
n \\
i
\end{array}\right)(p \mu)^{i} \\
& =\frac{\underline{\lambda}}{\lambda-\mu}(1+p \lambda)^{n}-\frac{\underline{\mu}}{\lambda-\mu}(1+p \mu)^{n} \\
& =\frac{1}{\lambda-\mu}\left(\underline{\lambda} \lambda^{2 n}-\underline{\mu} \mu^{2 n}\right) \\
& =\mathcal{Q}_{2 n} .
\end{aligned}
$$

Theorem 2.5 For $n \geq 0$, we have

$$
\begin{aligned}
& \sum_{i=0}^{n}\left(\begin{array}{c}
n \\
2 i
\end{array}\right) \mathcal{Q}_{4 i}= \begin{cases}\frac{1}{2}\left(\Delta^{\frac{n}{2}}+p^{n}\right) \mathcal{Q}_{n} & \text { if } n \text { is even, } \\
\frac{1}{2}\left(\Delta^{\frac{n-1}{2}} \mathcal{K}_{n}-p^{n} \mathcal{Q}_{n}\right) & \text { if } n \text { is odd },\end{cases} \\
& \sum_{i=0}^{n}\left(\begin{array}{c}
n \\
2 i
\end{array}\right) \mathcal{K}_{4 i}= \begin{cases}\frac{1}{2}\left(\Delta^{\frac{n}{2}}+p^{n}\right) \mathcal{K}_{n} & \text { if } n \text { is even, } \\
\frac{1}{2}\left(\Delta^{\frac{n+1}{2}} \mathcal{Q}_{n}-p^{n} \mathcal{K}_{n}\right) & \text { if } n \text { is odd, }\end{cases} \\
& \sum_{i=0}^{n}\left(\begin{array}{c}
n \\
2 i+1
\end{array}\right) \mathcal{Q}_{4 i+1}= \begin{cases}\frac{1}{2}\left(\Delta^{\frac{n}{2}}-p^{n}\right) \mathcal{Q}_{n} & \text { if } n \text { is even, } \\
\frac{1}{2}\left(\Delta^{\frac{n-1}{2}} \mathcal{K}_{n}+p^{n} \mathcal{Q}_{n}\right) & \text { if } n \text { is odd },\end{cases} \\
& \sum_{i=0}^{n}\left(\begin{array}{c}
n \\
2 i+1
\end{array}\right) \mathcal{K}_{4 i+1}= \begin{cases}\frac{1}{2}\left(\Delta^{\frac{n}{2}}-p^{n}\right) \mathcal{K}_{n} & \text { if } n \text { is even }, \\
\frac{1}{2}\left(\Delta^{\frac{n+1}{2}} \mathcal{Q}_{n}+p^{n} \mathcal{K}_{n}\right) & \text { if } n \text { is odd } .\end{cases}
\end{aligned}
$$


Proof By Theorem 2.3, we obtain

$$
\begin{aligned}
\sum_{i=0}^{n}\left(\begin{array}{l}
n \\
2 i
\end{array}\right) \mathcal{Q}_{4 i} & =\frac{1}{2} \sum_{i=0}^{n}\left(\begin{array}{l}
n \\
i
\end{array}\right)\left(1+(-1)^{i}\right) \mathcal{Q}_{2 i} \\
& =\frac{1}{2}\left[\sum_{i=0}^{n}\left(\begin{array}{l}
n \\
i
\end{array}\right) \mathcal{Q}_{2 i}+\sum_{i=0}^{n}\left(\begin{array}{l}
n \\
i
\end{array}\right)(-1)^{i} \mathcal{Q}_{2 i}\right] \\
& = \begin{cases}\frac{1}{2}\left(\Delta^{\frac{n}{2}}+p^{n}\right) \mathcal{Q}_{n} & \text { if } n \text { is even, } \\
\frac{1}{2}\left(\Delta^{\frac{n-1}{2}} \mathcal{K}_{n}-p^{n} \mathcal{Q}_{n}\right) & \text { if } n \text { is odd. }\end{cases}
\end{aligned}
$$

Theorem 2.6 For $n \geq 0$, we have

$$
\begin{aligned}
& \sum_{i=0}^{n}\left(\begin{array}{l}
n \\
i
\end{array}\right)\left(\mathcal{Q}_{i}\right)^{2}= \begin{cases}\Delta^{\frac{n-2}{2}}\left(2 \mathcal{K}_{n}-\left(V_{5}+p\right) V_{n+1}-\left(V_{4}+V_{0}\right) V_{n}\right) & \text { if } n \text { is even, } \\
\Delta^{\frac{n-1}{2}}\left(2 \mathcal{Q}_{n}-\left(V_{5}+p\right) U_{n+1}-\left(V_{4}+V_{0}\right) U_{n}\right) & \text { if } n \text { is odd },\end{cases} \\
& \sum_{i=0}^{n}\left(\begin{array}{l}
n \\
i
\end{array}\right)\left(\mathcal{K}_{i}\right)^{2}= \begin{cases}\Delta^{\frac{n}{2}}\left(2 \mathcal{K}_{n}-\left(V_{5}+p\right) V_{n+1}-\left(V_{4}+V_{0}\right) V_{n}\right) & \text { if } n \text { is even, } \\
\Delta^{\frac{n+1}{2}}\left(2 \mathcal{Q}_{n}-\left(V_{5}+p\right) U_{n+1}-\left(V_{4}+V_{0}\right) U_{n}\right) & \text { if } n \text { is odd } .\end{cases}
\end{aligned}
$$

Proof From the quaternion multiplication, we have

$$
\begin{aligned}
& (\underline{\lambda})^{2}=2 \underline{\lambda}-\left(\left(V_{5}+p\right) \lambda+V_{4}+V_{0}\right), \\
& (\mu)^{2}=2 \underline{\mu}-\left(\left(V_{5}+p\right) \mu+V_{4}+V_{0}\right) .
\end{aligned}
$$

If we consider the Binet formula for the generalized Fibonacci quaternions, we have

$$
\begin{aligned}
\sum_{i=0}^{n}\left(\begin{array}{l}
n \\
i
\end{array}\right)\left(\mathcal{Q}_{i}\right)^{2} & =\sum_{i=0}^{n}\left(\begin{array}{l}
n \\
i
\end{array}\right)\left(\frac{\underline{\lambda} \lambda^{i}-\underline{\mu} \mu^{i}}{\lambda-\mu}\right)^{2} \\
& =\frac{1}{\Delta} \sum_{i=0}^{n}\left(\begin{array}{l}
n \\
i
\end{array}\right)\left[(\underline{\lambda})^{2} \lambda^{2 i}+(\underline{\mu})^{2} \mu^{2 i}-2 \underline{\lambda} \underline{\mu}(\lambda \mu)^{i}\right] \\
& =\frac{1}{\Delta} \sum_{i=0}^{n}\left(\begin{array}{l}
n \\
i
\end{array}\right)\left[(\underline{\lambda})^{2} \lambda^{2 i}+(\underline{\mu})^{2} \mu^{2 i}\right]-\frac{2 \underline{\lambda}}{\Delta} \sum_{i=0}^{n}\left(\begin{array}{l}
n \\
i
\end{array}\right)(-1)^{i} \\
& =\frac{(\underline{\lambda})^{2}}{\Delta} \sum_{i=0}^{n}\left(\begin{array}{l}
n \\
i
\end{array}\right) \lambda^{2 i}+\frac{(\underline{\mu})^{2}}{\Delta} \sum_{i=0}^{n}\left(\begin{array}{l}
n \\
i
\end{array}\right) \mu^{2 i} \\
& =\frac{(\underline{\lambda})^{2}}{\Delta}\left(1+\lambda^{2}\right)^{n}+\frac{(\underline{\mu})^{2}}{\Delta}\left(1+\mu^{2}\right)^{n} \\
& =\frac{(\underline{\lambda})^{2}}{\Delta}(\lambda \sqrt{\Delta})^{n}+\frac{(\underline{\mu})^{2}}{\Delta}(-\mu \sqrt{\Delta})^{n} \\
& =\Delta^{\frac{n-2}{2}}\left((\underline{\lambda})^{2} \lambda^{n}+(\underline{\mu})^{2}(-\mu)^{n}\right) \\
& =\left\{\begin{array}{l}
\Delta^{\frac{n-2}{2}}\left(2 \mathcal{K}_{n}-\left(V_{5}+p\right) V_{n+1}-\left(V_{4}+V_{0}\right) V_{n}\right) \quad \text { if } n \text { is even, } \\
\Delta^{\frac{n-1}{2}}\left(2 \mathcal{Q}_{n}-\left(V_{5}+p\right) U_{n+1}-\left(V_{4}+V_{0}\right) U_{n}\right) \quad \text { if } n \text { is odd. }
\end{array}\right.
\end{aligned}
$$

Note that, by taking $p=1$ in Theorem 2.6 , we obtain

$$
\sum_{i=0}^{n}\left(\begin{array}{l}
n \\
i
\end{array}\right)\left(Q_{i}\right)^{2}= \begin{cases}5^{\frac{n-2}{2}}\left(2 K_{n}-L_{n}-4 L_{n+4}\right) & \text { if } n \text { is even, } \\
5^{\frac{n-1}{2}}\left(2 Q_{n}-F_{n}-4 F_{n+4}\right) & \text { if } n \text { is odd }\end{cases}
$$


and

$$
\sum_{i=0}^{n}\left(\begin{array}{l}
n \\
i
\end{array}\right)\left(K_{i}\right)^{2}= \begin{cases}5^{\frac{n}{2}}\left(2 K_{n}-L_{n}-4 L_{n+4}\right) & \text { if } n \text { is even, } \\
5^{\frac{n+1}{2}}\left(2 Q_{n}-F_{n}-4 F_{n+4}\right) & \text { if } n \text { is odd }\end{cases}
$$

where $Q_{i}$ and $K_{i}$ are the $i$ th Fibonacci quaternion and the $i$ th Lucas quaternion, respectively.

\section{Competing interests}

The authors declare that they have no competing interests.

\section{Authors' contributions}

All authors typed, read, and approved the final manuscript.

\section{Acknowledgements}

The authors would like to thank the referees for their valuable suggestions and comments.

Received: 16 March 2015 Accepted: 18 May 2015 Published online: 05 June 2015

\section{References}

1. Vajda, S: Fibonacci and Lucas Numbers and the Golden Section. Ellis Horwood, Chichester (1989)

2. Carlitz, L: Some classes of Fibonacci sums. Fibonacci Q. 16, 411-426 (1978)

3. Carlitz, L, Ferns, HH: Some Fibonacci and Lucas identities. Fibonacci Q. 8, 61-73 (1970)

4. Hoggatt, VE: Some special Fibonacci and Lucas generating functions. Fibonacci Q. 9, 121-133 (1971)

5. Layman, JW: Certain general binomial-Fibonacci sums. Fibonacci Q. 15, 362-366 (1977)

6. Andrews, GE, Askey, R, Roy, R: Special Functions. Encyclopedia of Mathematics and Its Applications, vol. 71. Cambridge University Press, Cambridge (1999)

7. Araci, S, Agyuz, E, Acikgoz, M: On a q-analog of some numbers and polynomials. J. Inequal. Appl. 2015, 19 (2015)

8. Graham, RL, Knuth, DE, Patashnik, O: Concrete Mathematics. Addison-Wesley, Reading (1994)

9. Kilic, E: Sums of the squares of terms of sequence $\left\{u_{n}\right\}$. Proc. Indian Acad. Sci. Math. Sci. 118, 27-41 (2008)

10. Kim, T, Dolgy, DV, Kim, DS, Rim, S-H: A note on the identities of special polynomials. Ars Comb. 113A, 97-106 (2014)

11. Simsek, Y: Special functions related to Dedekind-type DC-sums and their applications. Russ. J. Math. Phys. 17, 495-508 (2010)

12. Srivastava, HM, Choi, J: Zeta and q-Zeta Functions and Associated Series and Integrals. Elsevier, Amsterdam (2012)

13. Horadam, AF: Complex Fibonacci numbers and Fibonacci quaternions. Am. Math. Mon. 70, 289-291 (1963)

14. Ramirez, J: Some combinatorial properties of the k-Fibonacci and the k-Lucas quaternions. An. Ştiinţ. Univ. 'Ovidius' Constanţa, Ser. Mat. 23(2), 201-212 (2015)

15. lakin, AL: Extended Binet forms for generalized quaternions of higher order. Fibonacci Q. 19, 410-413 (1981)

16. Iyer, MR: A note on Fibonacci quaternions. Fibonacci Q. 3, 225-229 (1969)

17. Swamy, MNS: On generalized Fibonacci quaternions. Fibonacci Q. 5, 547-550 (1973)

18. lakin, AL: Generalized quaternions of higher order. Fibonacci Q. 15, 343-346 (1977)

19. lakin, AL: Generalized quaternions with quaternion components. Fibonacci Q. 15, 350-352 (1977)

20. Horadam, AF: Quaternion recurrence relations. Ulam Q. 2, 23-33 (1993)

21. Halici, S: On Fibonacci quaternions. Adv. Appl. Clifford Algebras 22, 321-327 (2012)

22. Flaut, C, Shpakivskyi, V: On generalized Fibonacci quaternions and Fibonacci-Narayana quaternions. Adv. Appl. Clifford Algebras 23, 673-688 (2013)

\section{Submit your manuscript to a SpringerOpen ${ }^{\circ}$ journal and benefit from:}

- Convenient online submission

Rigorous peer review

- Immediate publication on acceptance

Open access: articles freely available online

- High visibility within the field

- Retaining the copyright to your article 